\title{
On the Design of a WiFi Direct 802.11ac WLAN under a TGn MI MO Multipath Fading Channel
}

\author{
Gul Zameen Khan ${ }^{1}$, Ruben Gonzalez ${ }^{2}$, and Eun-Chan Park ${ }^{3}$ \\ ${ }^{1,2}$ School of ICT, Griffith University, \\ Gold Coast, 4215, Australia \\ [e-mail: ${ }^{1}$ gz.khan@griffithuni.edu.au, ${ }^{2}$ r.gonzalez@griffith.edu.au] \\ ${ }^{3}$ Department of Information and Communication Engg., Dongguk University, \\ Seoul, South Korea \\ [e-mail: ecpark@dongguk.edu ${ }^{3}$ ] \\ *Corresponding author: Gul Zameen Khan
}

Received July 21, 2016; revised November 5, 2016; revised December 15, 2016; accepted December 27, 2016; published March 31, 2017

\begin{abstract}
WiFi Direct (WD) is a state of the art technology for a Device-to-Device (D2D) communication in 802.11 networks. The performance of the WD system can be significantly affected by some key factors such as the type of application, specifications of MAC and PHY layer parameters, and surrounding environment etc. It is, therefore, important to develop a system model that takes these factors into account. In this paper, we focus on investigating the design parameters of the PHY layer that could maximize the efficiency of the WD 802.11 system. For this purpose, a basic theoretical model is formulated for a WD network under a 2x2 Multiple In Multiple Out (MIMO) TGn channel B model. The design level parameters such as input symbol rate and antenna spacing, as well as the effects of the environment, are thoroughly examined in terms of path gain, spectral density, outage probability and Packet Error Rate (PER). Thereafter, a novel adaptive algorithm is proposed to choose optimal parameters in accordance with the Quality of Experience (QoE) for a targeted application. The simulation results show that the proposed method outperforms the standard method thereby achieving an optimal performance in an adaptive manner.
\end{abstract}

Keywords: Device-to-Device, WiFi Direct, design parameters, system model, PHY layer, MIMO TGn channel B, adaptive, optimized, Quality of Experience 


\section{Introduction}

In recent years, Device-to-Device (D2D) communication has emerged as one of the most promising technologies in popular wireless networks (i.e., cellular networks, Wi-Fi, Bluetooth, and, Zigbee etc). The D2D communication has several advantages such as increased spectral efficiency, high throughput, and reduced communication delay [1]. In Wireless Local Area Networks (WLAN) 802.11, the D2D communication is achieved with the help of WiFi Direct (WD) technology [2]. WD has a wide range of applications including but not limited to content sharing, network services sharing, local multimedia applications, video games, and proximity and location based services etc. [3].

The WD technology was developed by the WiFi alliance [2]. It enables easy, quick, and direct communication between two or more nearby devices in the absence of an Access Point (AP). Point-to-point connectivity was already present in IEEE 802.11 standard [4] as an ad-hoc network. However, this did not support device/service discovery and efficient power saving techniques. Similarly, Tunneled Direct Link Setup (TDLS) also allowed two WiFi devices to connect directly with each other [5]. However, this requires all the devices that want to communicate with each other to be associated with the same AP. In contrast, there are no such restrictions in WD, making it the de facto technology for D2D communication in WiFi networks.

IEEE 802.11ac [6] is the latest standard in the 802.11 families and has been adopted by most new WiFi products [7]. The 802.11ac standard achieves Very High Throughput (VHT) due to a number of new features such as increased channel bandwidth, higher Modulation and Coding Schemes (MCSs), and advanced Medium Access Channel (MAC) techniques. When a WD network incorporates the MAC and Physical (PHY) layers specifications of the 802.11ac standard, we refer to it as a WD 802.11ac network.

Current literature on WD focuses on improving its high-level architecture and applications. This includes fast group formation, efficient power saving methods, and applications of WD in cellular data offloading etc. In this regard, Camps-Mur et al. [8] presented an overview of the architecture and group formation methods for WD. Therein, the delays of the three main group formation methods in WD were experimentally compared. However, the authors did not consider the underlying technology (i.e., PHY layer and wireless channel) in their experiment. Similarly, in [9] mobile data offloading onto network assisted D2D links between nearby user devices was investigated in which an analytical methodology and system level simulation were developed. The results showed an increased wireless capacity gain, improved degree of spatial reuse, and smaller interference. The authors in [10] presented a Gilbert-Elliot model to analyze the performance of a loss model of a wireless channel between two mobile phones using the WD technology. However, the experiment was performed for User Datagram Protocol (UDP) packets without taking into consideration the PHY layer parameters. Similarly, [11] evaluated the outage probability of a WD network under a Rayleigh fading environment for smart grid applications. The network was modeled as a Markov chain process and it was shown that the traffic intensity and the number of active devices could have a significant impact on the capacity in power save mode. However, this model assumes a general fading wireless channel, which is not directly applicable to WiFi or WD environment. In the same way, Xiaofeng et al. [12] investigated disruptions between mobile terminals due to user mobility. A Subscribe-and-Send architecture with an opportunistic forwarding protocol was proposed to share the contents among P2P users. Nonetheless, the protocol considers only 
the application layer without taking into account the effects of the channel and other lower layers.

The WiFi alliance set a general rule that the Quality of Service (QoS) of WD is based on the QoS of the standard WiFi [7]. For example, the problem of unfairness among WD enabled devices can be solved by different approaches already present in the literature. One such approach is [13], which achieves per-device fairness and service differentiation using MAC layer measurements such as the aggregate value of network-wide traffic load, per-device link usage, and per-device link error rate. In the same way, new concepts of resource allocation using relays from cellular communication [14] can be used to enhance the QoS of a WD. However, it is important for the integration of the QoS algorithms from standard WiFi, as well as, cellular networks, to perform a detailed analysis of the underlying layers of the WD and the environment in which the communication takes place. Similarly, W. H. Kuo et al. present a Quality of Experience (QoE)-based link adaptation scheme for H.264/Scalable Video Coding (SVC) video streaming over IEEE $802.11 \mathrm{~b} / \mathrm{g}$ wireless LANs [15].

It is also an established fact that most of the new wireless devices use Multiple In Multiple Out (MIMO) technologies to minimize errors and maximize capacity. The authors in [16] present a comprehensive overview of the basic concepts of massive MIMO, and explain its challenges and opportunities, and explore contemporary research. Similarly, MU massive MIMO is also an emerging wireless technology wherein the deployment of large antennas in an efficient way is yet another practical challenge. For more details on MU massive MIMO communication systems using irregular antenna arrays, interested readers are encouraged to see [17].

There is a considerable amount of research on the effects of roving transmitters and receivers in WiFi. However, in this paper, we consider a typical office environment, having stationary transmitters and receivers within a dynamic physical environment. People moving around in the office, can substantially affect the ongoing wireless communication. Thus, we concentrate on the effects of the movement of surrounding objects instead of the transmitter and receiver.

As WD 802.11ac has several potential applications in a small office environment, it becomes important to explore the performance of WD 802.11ac networks under MIMO multipath fading channels. To this end, our paper makes the following contributions:

1) We formulate a theoretical system model that is closely related to the real world network scenario (i.e., WD 802.11ac network under a 2x2 MIMO multipath fading TGn channel B) in a typical office or residence.

2) We explore the effects of different PHY layer parameters as well as the effects of the surrounding environment on the performance of WD 802.11ac network under a 2x2 MIMO multipath fading TGn channel B.

3) We quantify the performance loss in terms of path gain and outage probability that can be used as an adaptive model to achieve a targeted performance for QoS provisioning.

4) A novel adaptive method is proposed to choose the PHY parameters that achieve an optimal performance in terms of QoE for a targeted application.

The remainder of the paper is organized as follows. In Section 2, a brief overview of the 802.11ac standard and WD technology is presented. Then, the system model is described in Section 3. Next, the simulation environment and results are discussed in Section 4. Finally, conclusions are drawn in Section 5. 


\section{Background}

In this section, we briefly describe the main features of the underlying technologies (i.e., 802.11ac and the WD).

\subsection{IEEE 802.11ac}

Compared to IEEE 802.11a/g/n, the new 802.11ac variant supports higher MCS such as 256-Quadrature Amplitude Modulation (QAM) with a coding rate of 5/6. Similarly, the number of spatial streams or antennas is increased from 4 in 802.11n [18] up to 8 in 802.11ac at the PHY layer in Orthogonal Frequency Division Multiplexing (OFDM)-MIMO systems. In addition, with MU-MIMO in 802.11ac, it is possible to stream multiple data frames from an AP to multiple receivers at the same time [6]. Previous IEEE standards, such as $802.11 \mathrm{a} / \mathrm{g} / \mathrm{n}$ have overcrowded the unlicensed band of $2.4 \mathrm{GHz}$. Thus, 802 .11ac operates only in the $5 \mathrm{GHz}$ band thereby decreasing interference in the $2.4 \mathrm{GHz}$ band. The $802.11 \mathrm{ac}$ standard provides increased bandwidth through channel aggregation techniques. As such, it supports a range of channel widths (i.e., $20 \mathrm{MHz}, 40 \mathrm{MHs}, 80 \mathrm{MHz}$ and $160 \mathrm{MHz}$ ) wherein a $40 \mathrm{MHz}$ channel is achieved by aggregating two $20 \mathrm{MHz}$ either contiguous or non-contiguous channels. In the same way, $80 \mathrm{MHz}$ and $160 \mathrm{MHz}$ channels are obtained from aggregations of smaller channels.

\subsection{WiFi Direct (WD)}

WD is also known as Peer-to-Peer (P2P) WiFi [2]. In WD, all communication takes place in a group which is called a P2P group. Generally, any two or more devices with WiFi cards and at least one with a WD capability can make a group and communicate with each other. In every P2P group, there is one P2P Group Owner (GO) and one or more client devices.Thus when two or more devices intend to start a D2D communication using WD protocol, they must first form a P2P group. For the sake of simplicity, we will use the terms group and GO for a P2P group and P2P Group Owner, respectively in the rest of the paper.

\subsubsection{P2P Group Formation}

In group formation, a negotiation is undertaken to determine which device will act as a $G O$ and which device(s) will be a client(s). The GO must be a P2P WiFi device while the client can either be a P2P WiFi device or a regular WiFi device ${ }^{1}$. The role of the $G O$ is similar to the role of an AP. It transmits beacon frames that contain P2P Information Elements (IEs).

The $G O$ is required to run a Dynamic Host Configuration Protocol (DHCP) server to provide IP addresses to the clients. The group can cross connect to another external network (i.e., a 3G or an infrastructure network) through its GO. In addition, no bridging is allowed for cross connection. Therefore, the transfer is done at the network layer. However, a group can be extended by a P2P client to connect to another group. Similarly, the role of a $G O$ cannot be transferred within the group. The group is torn down if the GO leaves the group. Furthermore, the devices in a group can communicate with each other for a single session or they can retain the memory of the session and link together each time they come into proximity, which is called a Persistent group. In addition, the group formation also aims to exchange Credentials (i.e., the information that is required to join a group as defined by WPS [19] for a group). A group can be formed in any one of the three ways namely: standard, persistent and

\footnotetext{
${ }^{1}$ A P2P WiFi device is any device with WiFi card that also supports WD technology while a regular WiFi device is any device with only WiFi 802.11 protocol.
} 
autonomous group formation methods. Once the group is formed, the $G O$ and client(s) are ready to exchange data. For more details on group formation, refer to [2].

\subsubsection{Service Discovery and Power Management}

Service discovery is supported at layer 2 and can be used for many useful applications. This includes the advertisement of higher layer services such as Bonjour, UPnP (Universal Plug and Play), and Web Service Discovery [20]. WD also provides power management mechanisms for reducing power consumption for all devices within a group while maintaining discovery capabilities. The realization of power savings, however, will depend on the settings of and interaction between devices in a given environment.

\subsubsection{Example Scenario}

There are a number of scenarios where WD technology is the most efficient and easiest option for D2D communication. For example: sharing data files between the devices that are in close proximity to each other, sharing services such as an office printer, streaming media in a home/office environment etc. One user case scenario is that of a speaker delivering a presentation during an office meeting. This is depicted in Fig. 1. where the presenter shares the presentation slides from his/her laptop and displays them on a TV or LCD screen using WD technology (The image in Fig. 1 has been captured from an animated video in [21] and has been modified for our purpose.). In order to develop a system model that closely mimics a real application in a small office environment, our model consists of one $G O$ and one client with the laptop being the client while the LCD screen is the GO.

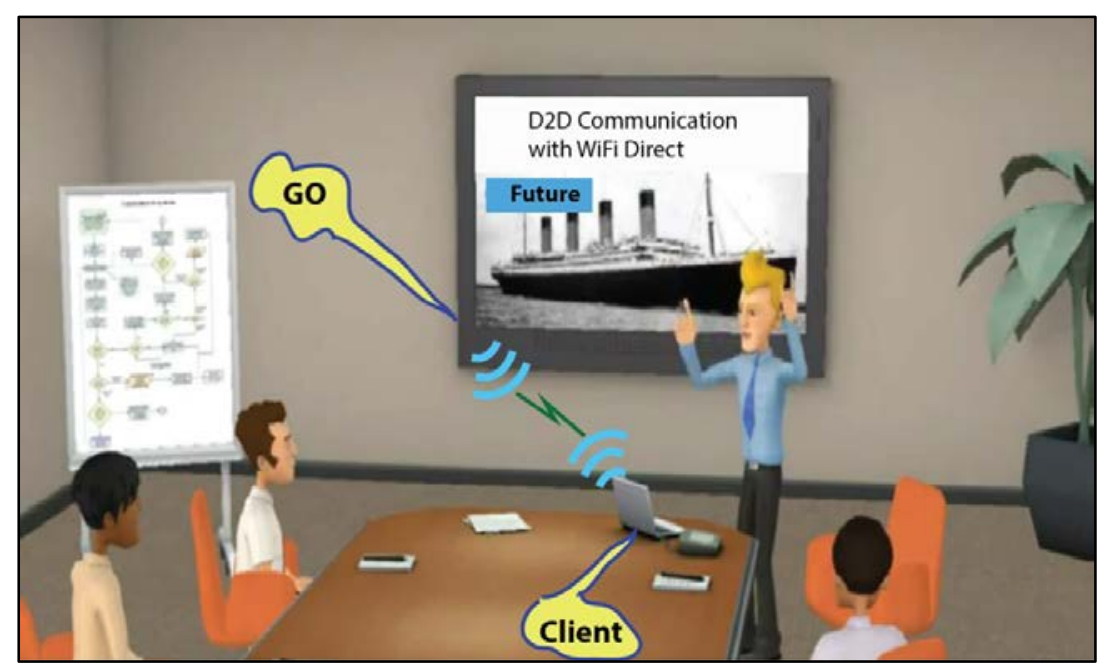

Fig. 1. WD network in a typical office

\section{System Model}

\subsection{Model Description}

We develop a system model for the PHY layer of a WD 802.11ac wireless network under a multipath flat fading MIMO channel. The system considers an OFDM-MIMO multipath fading channel to avoid frequency selective fading. A set of WLAN channels was presented in [22] for different environments. There are profiles proposed for channel models, labeled as 
TGn A to F [23]. These represent six different scenarios namely: flat fading, residential, residential/small office, typical office, large office, and large space (indoors and outdoors). Each channel model has a path loss model including shadowing, and a MIMO multipath fading model, which describes the multipath delay profile, the spatial properties, the K-factor distribution, and the Doppler spectrum.

We chose the TGn channel model B for our system so that the proposed system is applicable to the office environment as shown in Fig. 1. Further, we consider one $G O$ and one client. The client communicates with the GO using a WD protocol over an 802.11ac PHY layer. Both the $G O$ and the client have two antennas for transmission and reception. Thus, the channel is a 2x2 MIMO multipath fading TGn channel $\mathrm{B}$. As a general rule, this channel model can be extended to any number of clients, as well as, lower or higher order MIMOs. In addition, each channel in 802.11ac is characterized by a particular number of taps with respect to the first path delay where the overlapping subset of these tap delays makes a cluster. In the case of model B, there are two clusters; cluster 1 corresponding to tap delays 0 to 40 ns (in steps of 10 ns), while cluster 2 corresponding to tap delays 20 to 80 ns (also in steps of $10 \mathrm{~ns}$ ). Hence, cluster numbers 1 and 2 respectively comprise 5 and 7 tap delays, overlapping in 3 tap delays (20, 30 and $40 \mathrm{~ns})$. The number of taps, Root Mean Square (RMS) delay $\left(\sigma_{R M S}\right)$, maximum delay $\left(\sigma_{M a x}\right)$, the number of clusters and standard deviation of shadow fading in the case of both Line of Sight and Non-Line of Sight (NLOS) for each model are listed in Table 1.

Table 1. TGn channel B pathloss model 802.11ac

\begin{tabular}{|c|c|c|c|c|c|}
\hline Parameter & Value & Parameter & Value & Parameter & Value \\
\hline \hline K for LOS (dB) & 0 & Shadow fading before LOS (dB) & 3 & $\sigma_{R M S}(\mathrm{~ns})$ & 15 \\
\hline K for NLOS (dB) & $-\infty$ & Shadow fading after NLOS (dB) & 4 & $\sigma_{M a x}(\mathrm{~ns})$ & 80 \\
\hline Number of clusters & 2 & Number of taps & 9 & $d_{B P}(\mathrm{~m})$ & 5 \\
\hline
\end{tabular}

The system model is depicted in Fig. 2, where a client is working as a transmitter (Tx) and a $G O$ is the receiver $(\mathrm{Rx})$. Furthermore, the client (Tx) has two transmit antennas (i.e., $\mathrm{Tx}_{1}$ and $\mathrm{Tx}_{2}$ ) whereas the $G O(\mathrm{Rx})$ has two receive antennas namely: $\mathrm{Rx}_{1}$ and $\mathrm{Rx}_{2}$. The link between the transmit antenna $i\left(\mathrm{Tx}_{\mathrm{i}}\right)$ and the receive antenna $j\left(\mathrm{Rx}_{\mathrm{j}}\right)$ is indicated by $\mathrm{Tx}_{\mathrm{i}}-\mathrm{Rx}_{\mathrm{j}}$. We will use the same notations throughout this paper. However, for the sake of simplicity and due to space constraints, we have not shown all sub-blocks of the Tx and Rx. Also, only four blocks (i.e., digital modulation (Mod), Inverse Fast Fourier Transform (IFFT), pilot insertion, and Digital to Analog (D/A)) are illustrated. The diagram in Fig. $\mathbf{2}$ is used as a reference and for the sake of naming convenience. For a complete Tx and Rx diagrams with all the sub-blocks, we recommend [6].

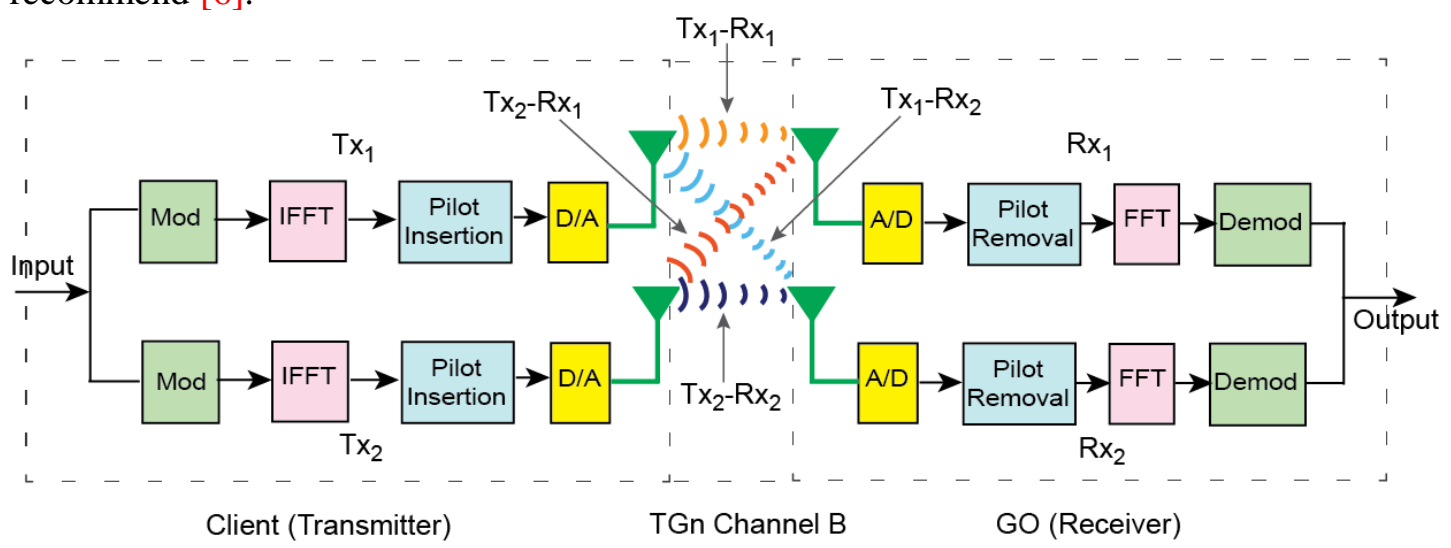

Fig. 2. A system model framework 


\subsection{Proposed System Model}

The multipath fading 2x2 MIMO channel of a WD 802.11ac network for TGn channel B is derived from the transmit and receive correlation matrices [24]. The transmit and receive correlation matrices associated with each tap delay are determined from the Mean Angle of Arrival (AoA), Mean Angle of Departure (AoD), Angular Spread (AS) at transmitter and AS at receiver. The LOS component can only be present on the 1st tap. If the distance between the Tx and the $\mathrm{Rx}$ is greater than $d_{B P}$ then LOS component is not present. Note that $d_{B P}$ is the break-point distance or distance of the fist wall (i.e., the distance of the Tx from the first reflector). The $d_{B P}$ for channel model B is listed in Table 1.

The path loss model considers the free space loss $L_{F S}$ (slope of 2) up to $d_{B P}$ and slope of 3.5 after $d_{B P}$ [25]. For each of the models, a different breakpoint distance $d_{B P}$ was chosen as shown in Eq. (1).

$$
L(d)= \begin{cases}L_{F S}(d)+\chi_{\sigma} & \text { if } d \leq d_{B P} \\ L_{F S}\left(d_{B P}\right)+35 \log 10\left(\frac{d}{d_{B P}}\right)+\chi_{\sigma} & \text { if } d>d_{B P}\end{cases}
$$

where $d$ shows the distance between the Tx and the $\mathrm{Rx}$ while $\chi_{\sigma}$ indicates a zero-mean, Gaussian random variable, with standard deviation, $\sigma$ (in $\mathrm{dB}$ ), added to the path loss. It is used to model the shadow fading, or log-normal shadowing. The values were found to be in the 3-14 $\mathrm{dB}$ range [26]. Similarly, the zero-mean Gaussian probability distribution is given by Eq. (2).

$$
p(x)=\frac{1}{\sqrt{2 \pi \sigma}} e^{\left(\frac{x^{2}}{2 \sigma^{2}}\right)}
$$

The 2x2 MIMO channel matrix $H$ for each tap, at one instance of time, under a channel B delay profile can be represented as a sum of two matrices namely: fixed (constant, LOS) matrix and a Rayleigh (variable, NLOS) matrix as illustrated in Eq. (3).

$$
H=\sqrt{P}\left(\sqrt{\frac{K}{K+1}} H_{F}+\frac{1}{K+1} H_{V}\right)
$$

where $P$ is the power of each tap obtained by summing all the power of LOS and NLOS powers, $K$ is the Ricean K-factor. The $H_{F}$ and $H_{V}$ in Eq. (3) can be extended for $2 \mathrm{x} 2 \mathrm{MIMO}$ as follows in Eq. (4-5):

$$
\begin{aligned}
H_{F} & =\left[\begin{array}{ll}
e^{\phi_{11}} & e^{\phi_{12}} \\
e^{\phi_{21}} & e^{\phi_{22}}
\end{array}\right] \\
H_{V} & =\left[\begin{array}{ll}
X_{11} & X_{12} \\
X_{21} & X_{22}
\end{array}\right]
\end{aligned}
$$

where $\mathrm{e}^{\phi_{i j}}$ shows the constant elements of LOS matrix $H_{F}$ whereas $X_{i j}$ represents the element of variable NLOS Rayleigh matrix $H_{V}$ between $i^{\text {th }}$ receiving and $j^{\text {th }}$ transmitting antenna. It is assumed that $X_{i j}$ is a complex Gaussian random variable with zero mean and unit variance.

In order to correlate the $X_{i j}$ elements of the matrix $H_{V}$, Eq. (6) is used.

$$
[X]=\left[R_{r x}\right]^{1 / 2} \times\left[H_{i i d}\right] \times\left[R_{t x}\right]^{1 / 2}
$$


where $\mathrm{R}_{\mathrm{tx}}$ and $\mathrm{R}_{\mathrm{rx}}$ are the receive and transmit correlation matrices, respectively, and $\mathrm{H}_{\mathrm{iid}}$ is a complex Gaussian random variable. All these Gaussian random variables are supposed to be identically independent with zero mean, unit variance. In addition, the $\mathrm{R}_{\mathrm{tx}}$ and $\mathrm{R}_{\mathrm{rx}}$ are given by Eq. (7) and Eq. (8), respectively.

$$
\begin{aligned}
& {\left[R_{t x}\right]=\left[\rho_{t x i j}\right]} \\
& {\left[R_{r x}\right]=\left[\rho_{r x i j}\right]}
\end{aligned}
$$

where $\rho_{\text {txij }}$ are the complex correlation coefficients between $\mathrm{i}^{\text {th }}$ and $\mathrm{j}^{\text {th }}$ transmitting antennas, and $\rho_{\text {rxij }}$ are the complex correlation coefficients between $\mathrm{i}^{\text {th }}$ and $\mathrm{j}^{\text {th }}$ receiving antennas.

Alternatively, we use another approach i.e., the Kronecker product of the transmit and receive correlation matrices Eq. (9) to calculate X.

$$
[X]=\left(\left[R_{t x}\right] \otimes\left[R_{r x}\right]\right)^{1 / 2} \times\left[H_{i i d}\right]
$$

It can be seen that $H_{i i d}$ is an array in this case instead of matrix. The $R_{t x}$ and $R_{r x}$ matrices are given in Eq. (10) and Eq. (11), respectively.

$$
\begin{aligned}
& R_{t x}=\left[\begin{array}{cc}
1 & \rho_{t \times 12}{ }^{*} \\
\rho_{t x 21} & 1
\end{array}\right] \\
& R_{r x}=\left[\begin{array}{cc}
1 & \rho_{r x 12}{ }^{*} \\
\rho_{r x 21} & 1
\end{array}\right]
\end{aligned}
$$

The values of complex correlation coefficient $\rho$ are calculated from power angular spectrum (PAS), AS, mean AoA, mean AoD and individual tap powers [23],[27]. Consequently, for the Uniform Linear Array (ULA), the complex correlation coefficient at the linear antenna array is expressed as shown in Eq. (12).

$$
\rho=R_{X X}(D)+j R_{X Y}(D)
$$

where $D=\frac{2 \pi d}{\lambda}$, and $R_{X X}$ and $R_{X Y}$ are the cross-correlation functions between the real parts and between the real part and imaginary part, respectively. The equations for $R_{X X}$ and $R_{X Y}$ are given in Eq. (13) and Eq. (14), respectively.

$$
\begin{aligned}
& R_{X X}(D)=\int_{-\pi}^{\pi} \cos \left(D \sin (\phi)_{-} P A S(\phi) d \phi\right. \\
& R_{X Y}(D)=\int_{-\pi}^{\pi} \sin \left(D \cos (\phi)_{-} P A S(\phi) d \phi\right.
\end{aligned}
$$

We calculate the correlation coefficients matrices using a uniform Gaussian PAS shape [23]. Once we have the channel matrix $\mathrm{H}$, we calculate the output symbol matrix $\mathrm{Y}$ given the input symbol matrix $\mathrm{X}$ as $\mathrm{Y}=\mathrm{HX}+\mathrm{W}$. The expression for a $2 \mathrm{x} 2 \mathrm{MIMO}$ system can be formulated as illustrated in Eq. (15) [28].

$$
\left[\begin{array}{l}
y_{1} \\
y_{2}
\end{array}\right]=\left[\begin{array}{ll}
h_{11} & h_{12} \\
h_{21} & h_{22}
\end{array}\right]\left[\begin{array}{l}
x_{1} \\
x_{2}
\end{array}\right]+\left[\begin{array}{l}
w_{1} \\
w_{2}
\end{array}\right]
$$


where $y_{1}$ and $y_{2}$ are the output symbols received at antennas $\mathrm{Rx}_{1}$ and $\mathrm{Rx}_{2}$ respectively. Similarly, $w_{1}$ and $w_{2}$ are the noise components at antennas $\mathrm{Rx}_{1}$ and $\mathrm{Rx}_{2}$ respectively, whereas $x_{1}$ and $x_{2}$ are the input symbols transmitted from antennas $\mathrm{Tx}_{1}$ and $\mathrm{Tx}_{2}$ respectively. In the same way, $h_{i j}$ is the channel coefficient for link $\mathrm{Tx}_{\mathrm{i}}-\mathrm{Rx}_{\mathrm{j}}$.

Now we calculate the expected average Signal to Noise Ratio (SNR) at the GO based on the pathloss model defined in Eq. (1) for TGn Channel B. We assume that the client transmits with a transmit power of $5 \mathrm{dBm}$, and the receive sensitivity (or signal detection threshold) at the $G O$ is $-90 \mathrm{dBm}$. Furthermore, we ignore the presence of any other external interference. Thereafter, the SNR values for different distance values $d$ between the $G O$ and client are calculated as illustrated in Table 2.

Table 2. SNR values for diffrenet distances for a TGn channel B

\begin{tabular}{|c||c|c|c|c|c|c|c|c|c|c|c|}
\hline $\mathbf{d}(\mathbf{m})$ & 1 & 3 & 6 & 9 & 12 & 15 & 21 & $\ldots$ & 30 & $\ldots$ & 46 \\
\hline SNR (dB) & 48 & 38.5 & 32.5 & 29 & 25 & 22 & 16 & $\ldots$ & 9 & $\ldots$ & 5 \\
\hline
\end{tabular}

The Quality of Experience (QoE) is defined as the overall acceptability of an application or service, as perceived subjectively by the end-user [29]. The QoE is a subjective measurement that includes the complete end-to-end system effects (client, terminal, network, services infrastructure). In order to assess the QoE, a Mean Opinion Score (MOS) is used. The MOS test specified by International Telegraph Union-Telecommunication (ITU-T) is shown in Table 3. We consider five types of applications (i.e., $A_{5}-A_{1}$ ) that run on our system. Each of these applications requires a different level of QoE.

Table 3. Mean Opinion Score

\begin{tabular}{|c|c|c|c|c|}
\hline \multirow{2}{*}{ MOS } & \multirow{2}{*}{ Quality } & Impairment & PER(\%) & Application \\
\hline \hline 5 & Excellent & Imperceptible & $0-20$ & $\mathrm{~A}_{5}$ \\
\hline 4 & Good & Perceptible but not annoying & $21-40$ & $\mathrm{~A}_{4}$ \\
\hline 3 & Fair & Slightly annoying & $41-60$ & $\mathrm{~A}_{3}$ \\
\hline 2 & Poor & Annoying & $61-80$ & $\mathrm{~A}_{2}$ \\
\hline 1 & Bad & Very annoying & $81-100$ & $\mathrm{~A}_{1}$ \\
\hline
\end{tabular}

While QoE is a subjective measurement that is highly dependent on a complex assortment of network, application and human factors, in this paper as a first order approximation we use PER in the ranges shown in Table 3 as a rough indicator of QoE such as [30]. Fixing the design parameters for the given system, so that the only parameters that can be chosen to adapt the performance of the system in accordance with the given QoE include MCS, SNR, and distance of transmitter and receiver. It permits the relationship between $S N R$ and distance $d$ be calculated from Eq. (1) and the Table 2 as shown in Eq. (16).

$$
d=f_{\text {dist }}(S N R)
$$

Now we formulate the adaptive optimized function $f_{A_{i}}($.$) to calculate the parameters that$ achieve the optimal QoE for a targeted application $\mathbf{A}_{\mathbf{i}}$ as shown in Eq. (16). These parameters include minimum SNR $\left(S N R_{\min }\right)$, maximum SNR $\left(S N R_{\max }\right)$, minimum distance between $G O$ and client $\left(d_{\min }\right)$, maximum distance between $G O$ and client $\left(d_{\max }\right)$, and MCS. 


$$
\text { Optimal paramters }=f_{A_{i}}\left(S N R_{\text {min }}, S N R_{\text {max }}, d_{\text {min }}, d_{\text {max }}, M C S\right) \forall \mathbf{A}_{\mathbf{i}} \text { and } \mathrm{MCS}_{\mathbf{j}}
$$

where $i=\{1,2,3,4,5\}$ and $j=\{0,1,2, \ldots, 8\}$ and MCS $_{\mathrm{j}}$ indicates one of the nine MCS defined for a 2x2 MIMO-OFDM WD system under a 20MHz bandwidth TGn B channel.

In order to calculate the optimal values, we define a matrix $R \in \mathbb{R}^{m \times n}$ that represents the range of SNR values for a where $m$ and $n$ indicate the number of applications and number of MCS, respectively. The element $R_{i j}$ indicates SNR range for application $\mathbf{A}_{\mathbf{i}}$ and $\mathrm{MCS}_{\mathrm{j}}$. We have calculated the values of $R_{i j}$ for application $\mathbf{A}_{\mathbf{i}}$ and MCS MCS ${ }_{\mathrm{j}}$ from our simulation setup as illustrated in Table. 5. The optimized values are calculated in Eq. (18-22).

$\mathbf{A}_{5}$ :

$$
\begin{gathered}
S N R_{\text {min }}=0, S N R_{\text {max }}=R_{1 j}-1, M C S=\mathrm{MCS}_{\mathrm{j}} \\
d_{\text {min }}=f_{\text {dist }}\left(S N R_{\text {min }}\right), d_{\text {max }}=f_{\text {dist }}\left(S N R_{\text {max }}\right)
\end{gathered}
$$

$\mathbf{A}_{4}$ :

$$
\left.\begin{array}{c}
S N R_{\text {min }}=R_{i j}, S N R_{\text {max }}=R_{1 j}+R_{2 j}-1, M C S=\mathrm{MCS}_{\mathrm{j}} \\
d_{\text {min }}=f_{\text {dist }}\left(S N R_{\text {min }}\right), d_{\text {max }}=f_{\text {dist }}\left(S N R_{\text {max }}\right)
\end{array}\right\}
$$

$\mathbf{A}_{3}$

$$
\left.\begin{array}{c}
S N R_{\text {min }}=R_{1 j}+R_{2 j}, S N R_{\text {max }}=R_{1 j}+R_{2 j}+R_{3 j}-1, M C S=\mathrm{MCS}_{\mathrm{j}} \\
d_{\text {min }}=f_{\text {dist }}\left(S N R_{\text {min }}\right), d_{\text {max }}=f_{\text {dist }}\left(S N R_{\text {max }}\right)
\end{array}\right\}
$$

$\mathbf{A}_{2}$

$$
\begin{gathered}
S N R_{\text {min }}=R_{1 j}+R_{2 j}+R_{3 j}, S N R_{\text {max }}=R_{1 j}+R_{2 j}+R_{3 j}+R_{4 j}-1, \\
M C S=\mathrm{MCS}_{\mathrm{j}}, d_{\text {min }}=f_{\text {dist }}\left(S N R_{\text {min }}\right), d_{\text {max }}=f_{\text {dist }}\left(S N R_{\text {max }}\right)
\end{gathered}
$$

$A_{1}$ :

$$
\begin{gathered}
S N R_{\text {min }}=R_{1 j}+R_{2 j}+R_{3 j}+R_{4 j}, \\
S N R_{\text {max }}=R_{1 j}+R_{2 j}+R_{3 j}+R_{4 j}+R_{5 j}-1, M C S=\mathrm{MCS}_{\mathrm{j}} \\
d_{\text {min }}=f_{\text {dist }}\left(S N R_{\text {min }}\right), d_{\text {max }}=f_{\text {dist }}\left(S N R_{\text {max }}\right)
\end{gathered}
$$

\section{Simulation Results and Discussions}

\subsection{Simulation Environment}

We have implemented the PHY layer of WD 802.11ac in Matlab with the given parameters as defined in [6], [23]. In order to implement MIMO channel B in NLOS mode, the physical parameters are used from Table 4 for clusters 1 and 2 in our simulation setup. As illustrated in Table 4, there are nine Rayleigh-fading paths, and each path has a bell Doppler spectrum with default parameters from [23]. 
Table 4. Parameters of TGn Channel B Model

\begin{tabular}{|c|c|c|c|c|c|c|c|c|c|c|}
\hline & Tap index & 1 & 2 & 3 & 4 & 5 & 6 & 7 & 8 & 9 \\
\hline & xcess Delay (ns) & 0 & 10 & 20 & 30 & 40 & 50 & 60 & 70 & 80 \\
\hline & Power (dB) & 0 & -5.4 & -10.8 & -16.2 & -21.7 & $-\infty$ & $-\infty$ & $-\infty$ & $-\infty$ \\
\hline \# & $\begin{array}{l}\text { AoA Tx } \\
\text { (degree) }\end{array}$ & 4.3 & 4.3 & 4.3 & 4.3 & 4.3 & $-\infty$ & $-\infty$ & $-\infty$ & $-\infty$ \\
\hline हैّ & AS (degree) & 14.4 & 14.4 & 14.4 & 14.4 & 14.4 & $-\infty$ & $-\infty$ & $-\infty$ & $-\infty$ \\
\hline 븡 & AoD (degree) & 255.1 & 255.1 & 255.1 & 255.1 & 255.1 & $-\infty$ & $-\infty$ & $-\infty$ & $-\infty$ \\
\hline & $\begin{array}{c}\text { AS Rx } \\
\text { (degree) }\end{array}$ & 14.4 & 14.4 & 14.4 & 14.4 & 14.4 & $-\infty$ & $-\infty$ & $-\infty$ & $-\infty$ \\
\hline & Power (dB) & $-\infty$ & $-\infty$ & -3.2 & -6.3 & -9.4 & -12.5 & -15.6 & -18.7 & -21.8 \\
\hline$N$ & $\begin{array}{l}\text { AoA Tx } \\
\text { (degree) }\end{array}$ & $-\infty$ & $-\infty$ & 118.4 & 118.4 & 118.4 & 118.4 & 118.4 & 118.4 & 118.4 \\
\hline 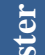 & AS (degree) & $-\infty$ & $-\infty$ & 25.2 & 25.2 & 25.2 & 25.2 & 25.2 & 25.2 & 25.2 \\
\hline U & AoD (degree) & $-\infty$ & $-\infty$ & 106.5 & 106.5 & 106.5 & 106.5 & 106.5 & 106.5 & 106.5 \\
\hline & $\begin{array}{c}\text { AS Rx } \\
\text { (degree) }\end{array}$ & $-\infty$ & $-\infty$ & 25.4 & 25.4 & 25.4 & 25.4 & 25.4 & 25.4 & 25.4 \\
\hline
\end{tabular}

\subsection{Results and Discussions}

In this section, we describe the impact of the design parameters (i.e., input symbol rate, adjacent antenna spacing of transmitter and receiver and Doppler shift) on the performance of WD 802.11ac networks under a 2x2 MIMO TGn B channel. Afterwards, the proposed method is applied to the given system.

\subsubsection{Effects of Input Symbol Rate}

IEEE 802.11ac uses OFDM to transmit the data over the air. The OFDM divides the available wider bandwidth (i.e., $20 \mathrm{MHz}, 40 \mathrm{MHz}, 80 \mathrm{MHz}$ or $160 \mathrm{MHz}$ ) into smaller slower channels that are also called subcarriers or tones. This process is achieved through a mathematical operation known as an Inverse Fast Fourier Transform (IFFT). All the subcarriers created by the IFFT in 802.11ac are $312.5 \mathrm{KHz}$ wide each. The Number of Subcarriers (NSC) is thus calculated as NSC = channel_Bandwith/(312.15*10 $\left.{ }^{3}\right)$ where channel_Bandwith indicates the bandwidth of the channel. The input symbol rate or baud rate for each subcarrier in the OFDM based WD 802.11ac network is defined as the number of symbols per unit time. The input symbol rate can greatly affect the impulse response in a wireless environment. To investigate the impact of a range of symbol rates, we take three different symbol rates (i.e., a low symbol rate $\left(10^{2}\right)$, a medium symbol rate $\left(10^{3}\right)$, and a higher symbol rate $\left(10^{4}\right)$ ).

As shown in Fig. 3(a), the fading envelope at antenna $\mathrm{Rx}_{1}$ for path 1 of link $\mathrm{Tx}_{1}-\mathrm{Rx}_{1}$ and link $\mathrm{Tx}_{2}-\mathrm{Rx}_{1}$ is unstable and highly cross-correlated at the lower symbol rate. On the other hand, when we increase the symbol rate to medium and higher levels as shown in Fig. 3(b-c), the probability of interference is reduced resulting in high performance. 


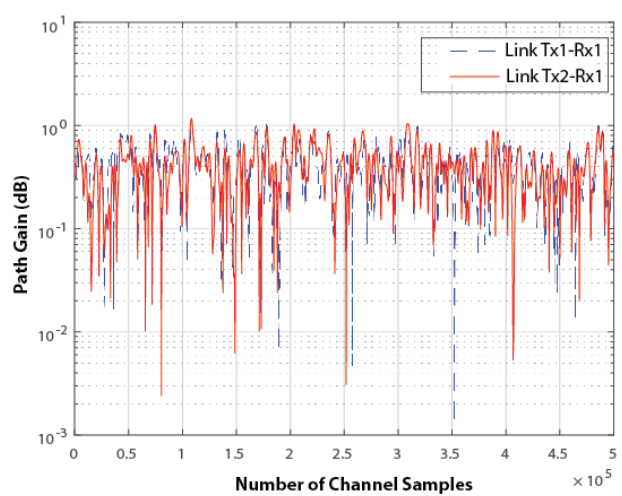

(a)

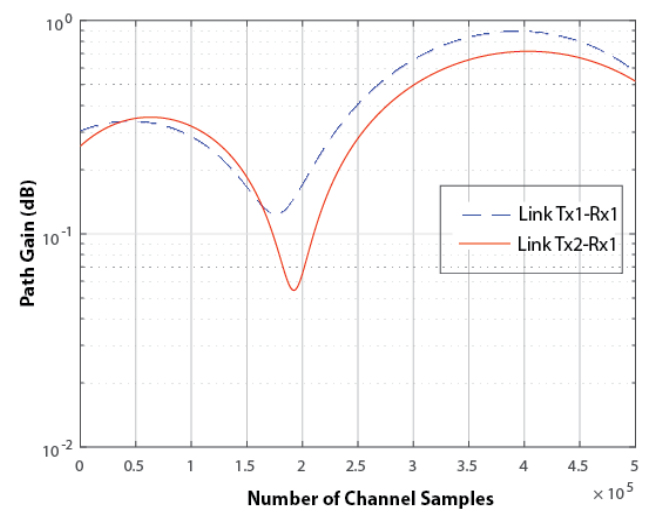

(c)

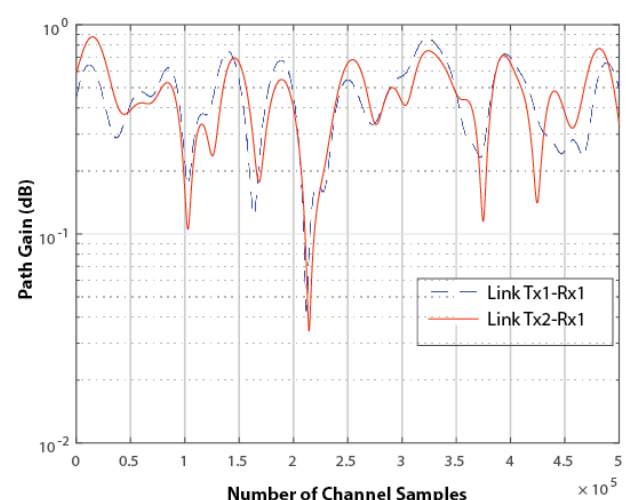

(b)

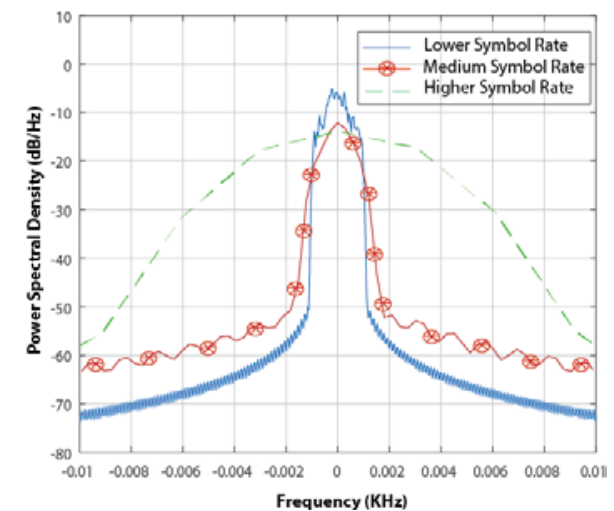

(d)

Fig. 3. Fading envelope and PSD for Links Tx1-Rx1 and Tx2-Rx1 with different symbol rate

Similarly, Fig. 3(d) represents the Power Spectral Density (PSD) for all the three levels of symbol rates along link $\mathrm{Rx}_{1}-\mathrm{Tx}_{1}$. The average PSD for the higher symbol rate is greater than that of the medium symbol rate. A similar trend can be seen for PSD of the medium symbol rate as compared to that of the lower symbol rate. Hence, increasing the input symbol rate can significantly increase the PSD.

\subsubsection{Effects of Adjacent Antenna Spacing}

In order to observe the impact of adjacent antenna spacing both at Tx and $\mathrm{Rx}$, we simulate the model with two different sets of antenna spacing (i.e., $\lambda / 2$ and $3 \lambda$ ). As shown in Fig. 4(a), there is a high cross-correlation between the signals at receiver antenna $\mathrm{Rx}_{1}$ received from transmitter antennas $\mathrm{Tx}_{1}$ and $\mathrm{Tx}_{2}$ when the adjacent antenna spacing is $\lambda / 2$. On the other hand, when the adjacent transmitter and receiver antenna spacing is increased to $3 \lambda$, the interference between the received signals is reduced as shown in Fig. 4(b).

Next, we repeat the simulations for different combinations of Tx and Rx antenna spacings and calculate the PER for different configurations. As shown in Fig. 5, the PER is 1 for all combinations of antenna spacings when the SNR is below $10 \mathrm{~dB}$. Similarly, the PER remains 0 for all configurations after $30 \mathrm{~dB}$ SNR. However, when SNR is between 10-30 dB, the PER drop is different for different Tx and Rx spacings. When the spacing between both Tx antennas and $\mathrm{Rx}$ antennas is increased from $\lambda / 2$ to $3 \lambda$, the PER is decreased by almost $10 \%$. Alternately, 
the PER is decreased by almost $11 \%$ when $\mathrm{Tx}_{1}-\mathrm{Tx}_{2}=3 \lambda$ and $\mathrm{Rx}_{1}-\mathrm{Rx}_{2}=\lambda / 2$ as compared to the case when $\mathrm{Tx}_{1}-\mathrm{Tx}_{2}=\lambda / 2$ and $\mathrm{Rx}_{1}-\mathrm{Rx}_{2}=3 \lambda$.

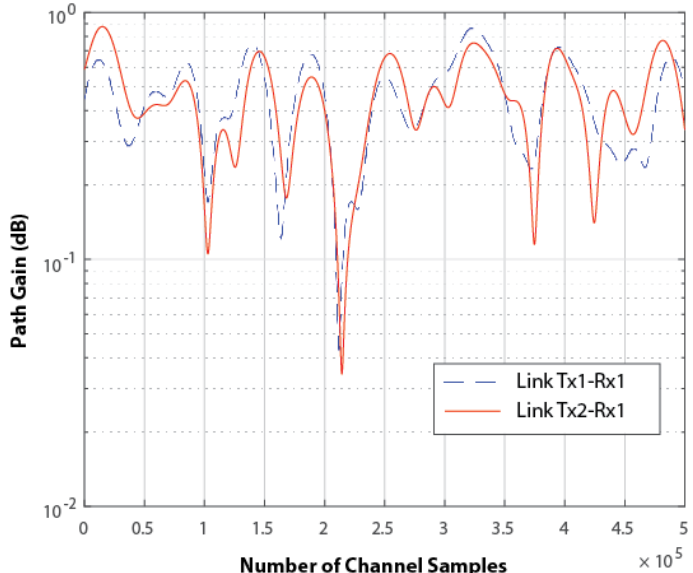

(a)

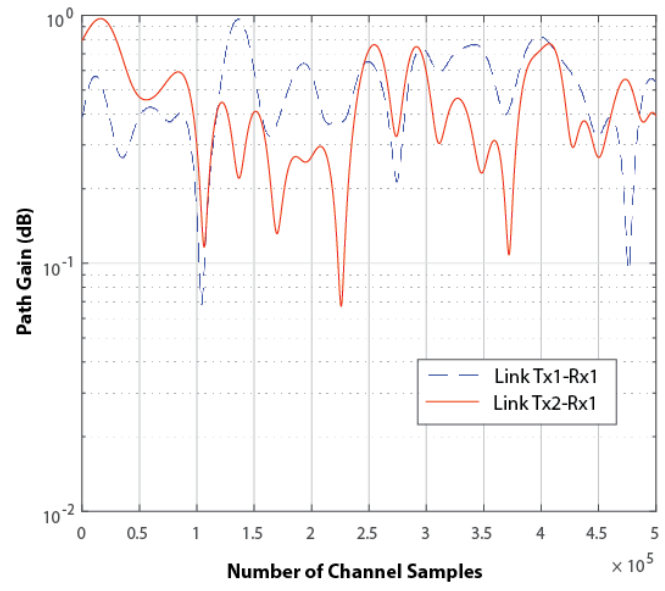

(b)

Fig. 4. Fading envelope for links $\mathrm{Tx}_{1}-\mathrm{Rx}_{1}$ and $\mathrm{Tx}_{2}-\mathrm{Rx}_{1}$ with antenna spacing of $0.5 \lambda$ and $3 \lambda$

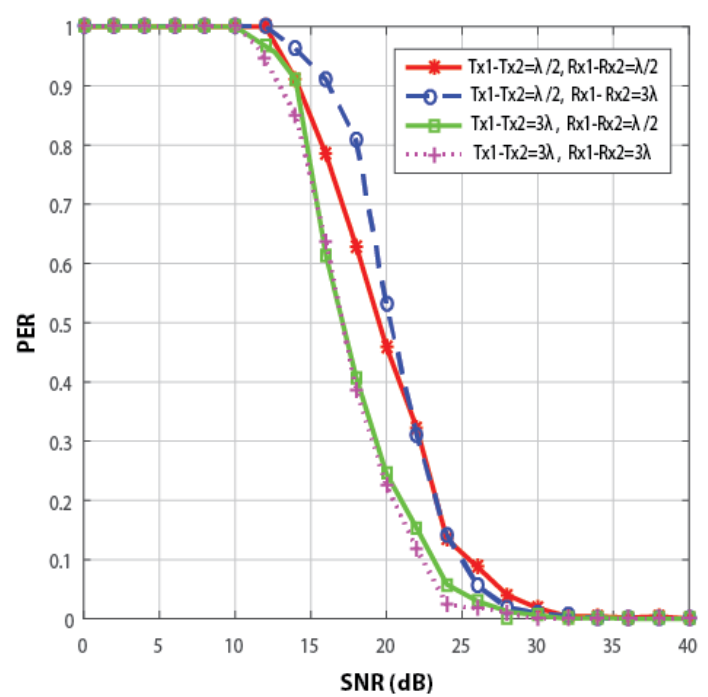

Fig. 5. PER for different configurations of Tx and Rx antenna spacings

\subsubsection{Effects of Doppler Shift}

As we are considering a TGn channel B that represents a typical small office environment, there is a reduced likelihood of Tx or Rx mobility relative to an outdoor environment. However, there is a high likelihood of other objects such as people moving within the environment. Therefore we use a function known as $S(f)$ to capture the temporal Doppler component. The normalized bell Doppler spectrum can be calculated from Eq. (23) that was derived in [22].

$$
S(f)=\frac{C_{b}}{1+A\left(\frac{f}{f_{d}}\right)^{2}}
$$


where $\mathrm{A}$ is a constant, used to define the $0.1 \mathrm{~S}(\mathrm{f})$, at a given frequency $f_{d}$, being the Doppler Spread.

$$
\left.(S(f))\right|_{f=f_{d}}=0.1
$$

Thus $A=0.9$. and $C_{b}=\frac{\sqrt{A}}{\pi f_{d}}$

The Doppler spread $f_{d}$ is defined in Eq. (25)

$$
f_{d}=\frac{v_{0}}{\lambda}
$$

where $v_{0}$ is the environmental speed determined from measurements that satisfy Eq. (23), and $\lambda$ is the wavelength defined by Eq. (26)

$$
\lambda=\frac{c}{f_{c}}
$$

where $c$ is the light speed and $f_{c}$ is the carrier frequency. The value for the proposed $v_{0}$ is equal to $1.2 \mathrm{~km} / \mathrm{h}$. In fact, $f_{d}$ values, experimentally determined in indoor environments, were found to be up to approximately $6 \mathrm{~Hz}$ at $5.25 \mathrm{GHz}$ center frequency.

In order to investigate the impact of moving objects on the performance of a WD 802.11ac network under a 2x2 MIMO TGn B channel, we calculate all possible Doppler shifts using a $5 \mathrm{GHz}$ carrier frequency. The Doppler shift turns out to be in the range of $1 \mathrm{~Hz}$ to $6 \mathrm{~Hz}$ for the TGn B channel model. Fig. 6(a)-(b) represent the fading envelope at antenna $\mathrm{Rx}_{2}$ for path 1 of link $\mathrm{Tx}_{1}-\mathrm{Rx}_{2}$ and link $\mathrm{Tx}_{2}-\mathrm{Rx}_{2}$. As shown in Fig. 6(a), the paths look more stable and there is less interference between the received signals in case of $1 \mathrm{~Hz}$ Doppler shift as compared to the same fading envelope where the Doppler shift is $6 \mathrm{~Hz}$, as shown in Fig. 6(b). Thus, the channel becomes worse when the Doppler shift changes from $1 \mathrm{~Hz}$ to $6 \mathrm{~Hz}$. Consequently, this reflects the movement of objects in the real world.

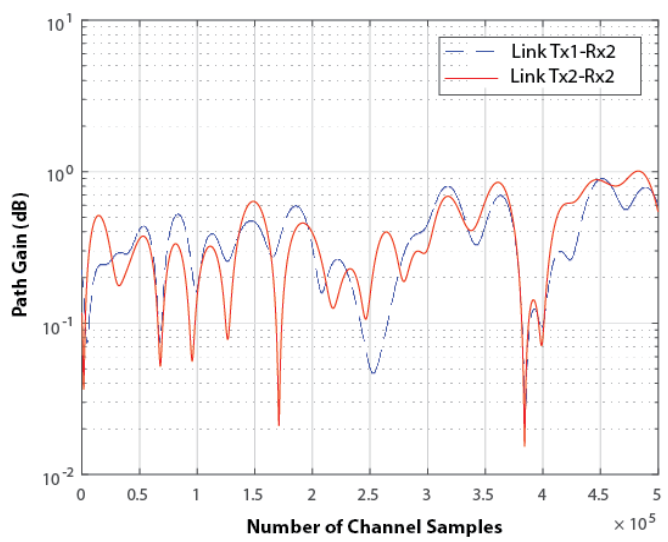

(a)

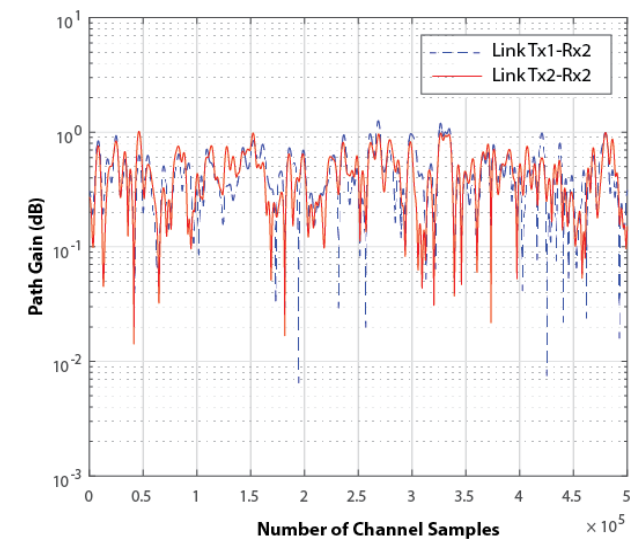

(b)

Fig. 6. Fading envelope for links Tx1-Rx2 and Tx2-Rx2 with Doppler shift of $1 \mathrm{~Hz}$ and $6 \mathrm{~Hz}$

We also calculate the outage probability $\left(P_{\text {out }}\right)$ of all links between a GO and client. It is calculated as shown in Eq. (27).

$$
P_{\text {out }}=\frac{\omega}{N_{\text {total }}}
$$

where $N_{\text {total }}$ is the total number of received channel samples, and $\omega$ is the cardinality of $N_{t h}$ 
received samples such that $N_{t h}=\left\{x \mid x \in\left[1, N_{\text {total }}\right] \wedge x<T h\right\}$. The $T h$ is the threshold power in $\mathrm{dB}$; its value is fixed at $-5 \mathrm{~dB}$ for our simulation. Table 5 illustrates the values of $P_{\text {out }}$ for all possible four links (i.e., $\mathrm{Tx}_{1}-\mathrm{Rx}_{1}, \mathrm{Tx}_{1}-\mathrm{Rx}_{2}, \mathrm{Tx}_{2}-\mathrm{Rx}_{1}$, and $\mathrm{Tx}_{2}-\mathrm{Rx}_{2}$ ). The $P_{\text {out }}$ increases as we increase the Doppler shift for all links. There is $38.53 \%$ loss when $f_{d}=1 \mathrm{~Hz}$, which rises to $46.67 \%$ for $f_{d}=6 \mathrm{~Hz}$. Furthermore, for $1 \mathrm{~Hz}$ increase in $f_{d}$, there is a loss of $1.56 \%$ on average. It is worth noting that the loss will decrease if the threshold $T h$ is decreased from $-5 \mathrm{~dB}$. Similarly, Fig. 7 represents the outage probability of all links with respect to Doppler shift. The $P_{\text {out }}$ for all links increases with an increase in $f_{d}$. This is because the frequency shift causes problems in detecting the original symbols at the $G O$.

Table 5. Outage probability of all links w.r.t Doppler shift

\begin{tabular}{|c|c|c|c|c|c|c|}
\hline \multirow{2}{*}{$\begin{array}{c}f_{d} \\
(\mathbf{H z})\end{array}$} & \multicolumn{3}{|c|}{$\boldsymbol{P}_{\text {out }}$ with Threshold $(\mathbf{T h}=-\mathbf{5 d B})$} & \multicolumn{2}{c|}{ Loss } \\
\cline { 2 - 7 } & $\mathbf{T x}_{\mathbf{1}}-\mathbf{R x}_{\mathbf{1}}$ & $\mathbf{T x}_{\mathbf{1}} \mathbf{-} \mathbf{R x}_{\mathbf{2}}$ & $\mathbf{T x}_{\mathbf{2}}-\mathbf{R} \mathbf{x}_{\mathbf{1}}$ & $\mathbf{T x}_{\mathbf{2}} \mathbf{-} \mathbf{R x}_{\mathbf{2}}$ & Average & \% \\
\hline \hline 1 & 0.3758 & 0.4042 & 0.3700 & 0.3912 & 0.3853 & 38.53 \\
\hline 2 & 0.4052 & 0.4337 & 0.3973 & 0.4248 & 0.4153 & 41.53 \\
\hline 3 & 0.4281 & 0.4539 & 0.4197 & 0.4513 & 0.4383 & 43.83 \\
\hline 4 & 0.4541 & 0.4643 & 0.4363 & 0.4620 & 0.4542 & 45.42 \\
\hline 5 & 0.4631 & 0.4681 & 0.4476 & 0.4739 & 0.4632 & 46.32 \\
\hline 6 & 0.4667 & 0.4676 & 0.4709 & 0.4507 & 0.4776 & 46.67 \\
\hline
\end{tabular}

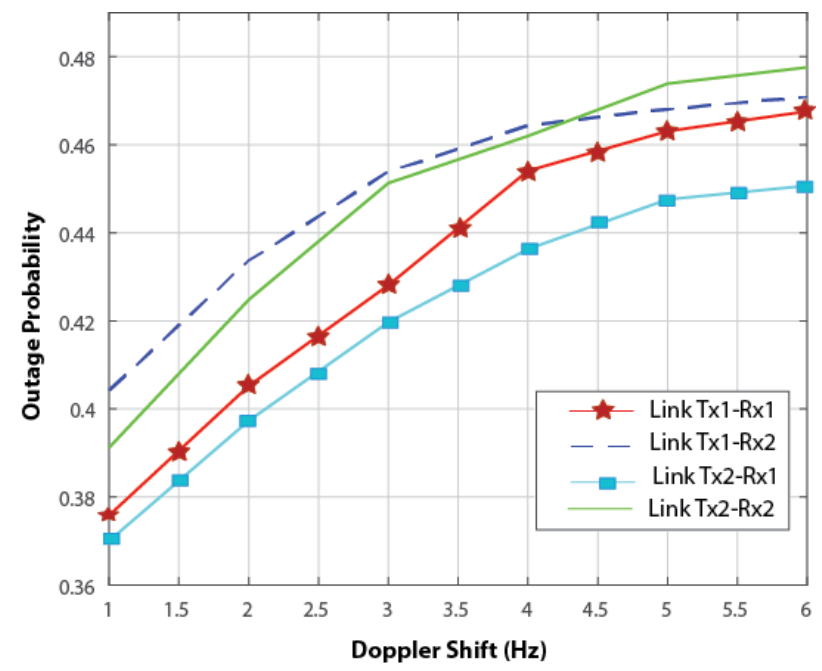

Fig. 7. Outage probability of all links with different Doppler shifts

\subsubsection{Adaptive Optimized QoE}

This section discusses the implementation of an QoE adaptation strategy for our system. As discussed in Sections 4.2.1 - 4.2.3, the design parameters (i.e., input symbol rate, the antenna spacing, as well as the effects of the environment in the form of Doppler shift) can greatly change the system performance. Thus, we propose a new adaptive optimized strategy to cope with such system degradation. Given our 2x2 MIMO-OFDM WD system with a $20 \mathrm{MHz}$ channel bandwidth under a TGn channel B, we calculate the PER for available $\mathrm{MCS}_{0}-\mathrm{MCS}_{8}$ as shown in Fig. 8. The PER for all MCSs decreases as we increase the SNR value, however, it is less for lower MCS as compared to higher MCS. 


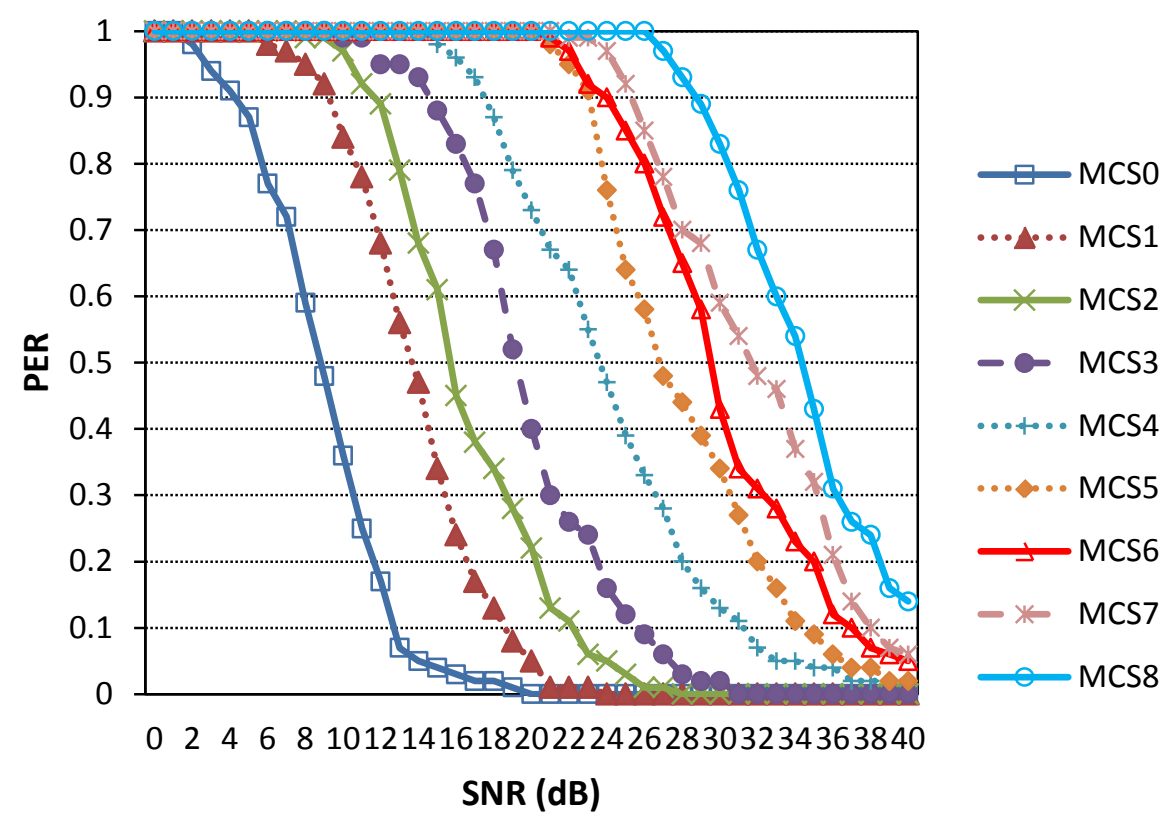

Fig. 8. PER for different MCS in 20MHz bandwidth

Table 5. Range (R) of SNR for different MCS under different applications

\begin{tabular}{|c|c|c|c|c|c|c|c|c|c|}
\hline $\begin{array}{c}\text { MCS } \\
\text { Application }\end{array}$ & $\mathbf{M C S}_{\mathbf{0}}$ & $\mathbf{M C S}_{\mathbf{1}}$ & $\mathbf{M C S}_{\mathbf{2}}$ & $\mathbf{M C S}_{\mathbf{3}}$ & $\mathbf{M C S}_{\mathbf{4}}$ & $\mathbf{M C S}_{\mathbf{5}}$ & $\mathbf{M C S}_{\mathbf{6}}$ & $\mathbf{M C S}_{\mathbf{7}}$ & $\mathbf{M C S}_{\mathbf{8}}$ \\
\hline \hline $\mathbf{A}_{\mathbf{5}}$ & 29 & 24 & 20 & 17 & 13 & 9 & 6 & 3 & 2 \\
\hline $\mathbf{A}_{\mathbf{4}}$ & 2 & 2 & 4 & 4 & 3 & 3 & 4 & 3 & 3 \\
\hline $\mathbf{A}_{3}$ & 2 & 2 & 1 & 1 & 2 & 3 & 2 & 5 & 3 \\
\hline $\mathbf{A}_{\mathbf{2}}$ & 2 & 2 & 3 & 2 & 4 & 2 & 3 & 3 & 2 \\
\hline $\mathbf{A}_{\mathbf{1}}$ & 6 & 11 & 13 & 17 & 19 & 24 & 26 & 27 & 31 \\
\hline
\end{tabular}

Let us now apply the proposed adaptive optimized method to the given system. We assume two scenarios. In scenario-1, the $G O$ and the client are $10 \mathrm{~m}$ apart while in scenario-2, they are $30 \mathrm{~m}$ apart. We calculate the MOS values for five different applications $\left(\mathrm{A}_{1}-\mathrm{A}_{5}\right)$ using the standard and our proposed method. The standard method refers to the regular WD 802.11 protocol [2] that chooses the MCS based on SNR values [4]. Since the implementation of the standard method may vary with different vendors, we choose the average value of PER obtained from all MCS for a particular SNR value. On the other hand, the proposed method adaptively chooses the optimized MCS and distance between the GO and the client based on the type of the application that in turns define a required QoE in terms of PER values as illustrated in Table 3. Consequently, we compare the performance of the standard and the proposed methods with an ideal case in terms of QoE values for a particular application.

\subsubsection{Scenario-1}

In this scenario, the $G O$ and client are placed $10 \mathrm{~m}$ apart. Thus $\mathrm{SNR}=28 \mathrm{~dB}$ for $d=10 \mathrm{~m}$ as per Table 2. Fig. 9 shows the QoE for the standard and proposed methods compared to the ideal case for five different applications $\left(\mathrm{A}_{5}-\mathrm{A}_{1}\right)$ with $\pm 5 \%$ error bar graphs. The QoE for the standard method remains 3.6 irrespective of the application. However, the proposed method achieves a QoE that is within 5\% of the ideal case. As shown in Fig. 9, the standard method 
results in degraded quality for $A_{5}$ and $A_{4}$ while wasting network resources in case of $A_{3}, A_{2}$, and $A_{1}$. In contrast, the proposed method optimally adapts its MCS resulting in a QoE which is quite close to the ideal case as shown by the $\pm 5 \%$ error bars.

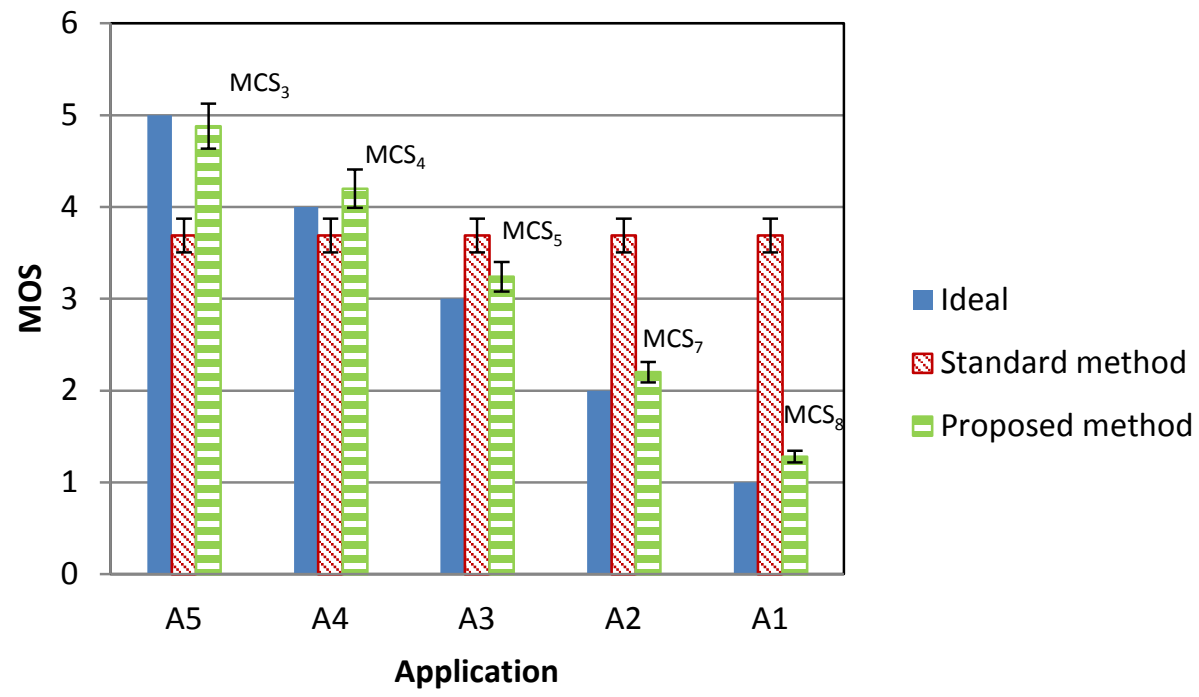

Fig. 9. QoE of five applications for the three methods for Scenario-1

\subsubsection{Scenario-2}

In the second scenario, the SNR=9dB at the $G O$ according to Table 2. The performance of the given system is calculated under both methods relative to the ideal case is shown in Fig. 10. We observe that the standard method achieves $\mathrm{QoE}=1.27$ for all the applications. In this case, the standard method performs better for $A_{1}$ but its performance substantially degrades for $A_{2}$, $A_{3}, A_{4}$, and $A_{5}$. In contrast, the proposed method achieves optimum performance by adapting an optimal distance $d$ of $21 \mathrm{~m}$ or equivalently SNR $=16 \mathrm{~dB}$ and chooses the appropriate MCSs in order to follow the ideal case as shown by \pm 7 error bars in Fig. 10.

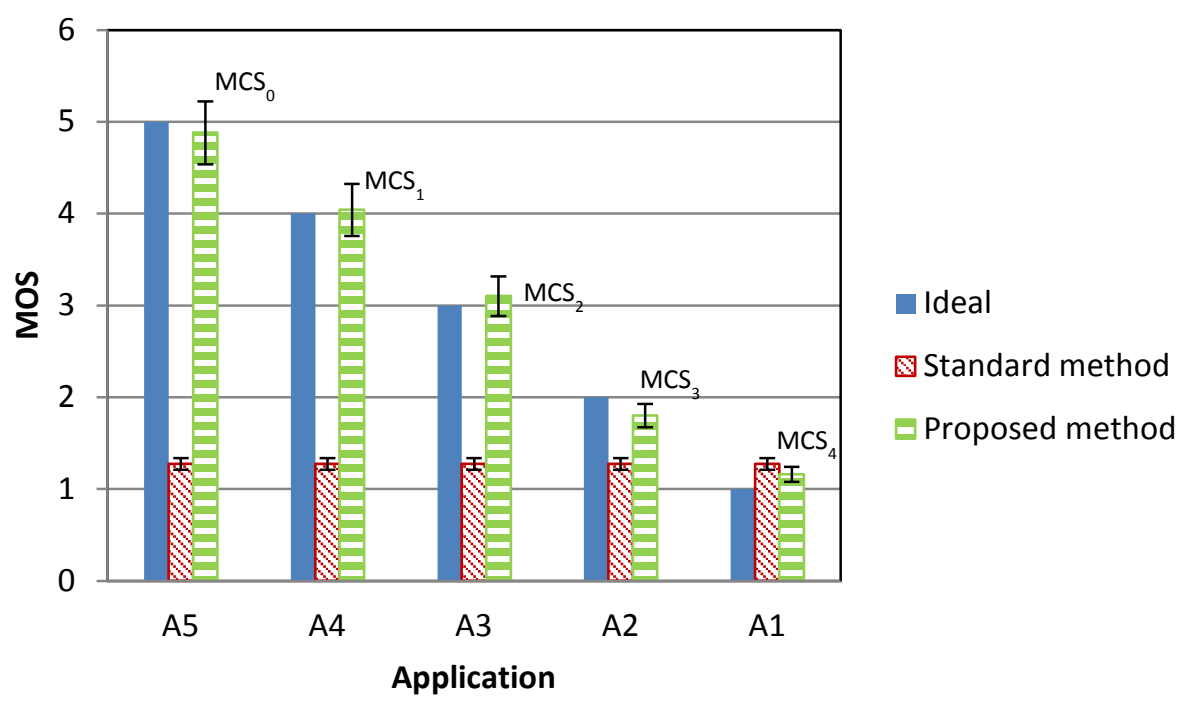

Fig. 10. QoE of five applications for the three methods for Scenaio-2 
In a nutshell, the proposed scheme improves the performance of a 2x2 MIMO-OFDM WD system with a $20 \mathrm{MHz}$ channel bandwidth under a TGn channel B. This is achieved by incorporating a novel QoE based algorithm that takes into account the key paramters such as MCS, PER, SNR, and the dstance $d$ between the GO and the client. The proposed method adaptibley chooses an optimized MCS and distance $d$ for five different applications. The results shown in Fig. 9 and Fig. 10 clearly illustrate that the proposed method outperforms the standard method. The two given scenarios are chosen such that a wide range of user cases can be imitated. In future work, the proposed method can also be extended to different seniors for other applications with different QoE requirements.

\section{Conclusion}

This paper investigated the design choices of a WD 802.11ac network under a MIMO multipath fading TGn B channel and thereafter proposed a novel adaptive algorithm that achieves optimal QoE for a particular application. To this end, a theoretical model was formulated for a 2x2 MIMO-OFDM under a TGn B channel. The design parameters such as input symbol rate and antenna spacing, as well as, the effects of the moving objects in the surrounding environment are examined for the given system. It was observed that the power of the received signal can be improved by almost $10 \mathrm{~dB}$ if the input symbol rate is increased from low symbol rate $\left(10^{2}\right)$ to a higher input symbol rate $\left(10^{4}\right)$. Similarly, the system performance can be enhanced up to $10 \%$ in terms of decreased PER if the spacing between two antennas at both the transmitter and receiver is increased from $\lambda / 2$ to $3 \lambda$. In addition, the investigations revealed that the loss due to the moving objects in the surrounding environment could substantially degrade the performance of the system that may reach up to $43.71 \%$ on average for a given threshold of the receiving power. Based on simulation results, a novel method is proposed that adapts the MCS, SNR, and the distance between the transmitter and the receiver in accordance with the required QoE for a targeted application. It was shown through simulation results that the proposed method performs much better than the standard method in choosing the PHY parameters.

\section{References}

[1] A. Asadi, W. Qing, and V. Mancuso, "A Survey on Device-to-Device Communication in Cellular Networks," IEEE Communications Surveys \& Tutorials, vol. 16, no. 4, pp. 801-1819, 2014. Article (CrossRef Link)

[2] Wi-Fi Alliance, “P2P Technical Group,” Wi-Fi Peer-to-Peer (P2P) Technical Specification v.1.0. 2009. Article (CrossRef Link)

[3] G. Z. Khan, R. Gonzalez, E. C. Park, and X. W. Wu, "A reliable multicast MAC protocol for Wi-Fi Direct 802.11 networks," in Proc. of IEEE European Conference on Networks and Communications (EuCNC) 2015, pp. 224-228, June 29, 2015. Article (CrossRef Link)

[4] IEEE 802.11-2007 Standard, Wireless LAN Medium Access Control (MAC) and Physical Layer (PHY) Specifications, IEEE 802.11-2007. Article (CrossRef Link)

[5] IEEE 802.11z-2010 Standard, Wireless LAN Medium Access Control (MAC) and Physical Layer (PHY) specifications Amendment 7: Extensions to Direct-Link Setup (DLS), IEEE 802.11-2010. Article (CrossRef Link)

[6] IEEE 802.11ac-2013 Standard, Enhancements for Very High Throughput for operation in bands below 6 GHz, IEEE P802.11ac/D5.0, 2013. Article (CrossRef Link) 
[7] Wi-Fi Alliance, WiFi-certified products finder. [Online]. Available: https://www.wi-fi.org/product-finder. Article (CrossRef Link)

[8] D. C. Mur, A. G. Saavedra, and P. Serrano, "Device-to-device communications with Wi-Fi Direct: overview and experimentation," IEEE Wireless Communications, vol. 20, no. 3, pp. 96-104, June, 2013. Article (CrossRef Link)

[9] S. Andreev, O. Galinina, A. Pyattaev, K. Johnsson, and Y. Koucheryavy, "Analyzing Assisted Offloading of Cellular User Sessions onto D2D Links in Unlicensed Bands," IEEE Journal on Selected Areas in Communications, vol.33, no.1, pp.67-80, January, 2015. Article (CrossRef Link)

[10] J. Feng, Z. Liu, and Y. Ji, "Wireless channel loss analysis - a case study using WiFi-Direct," in Proc. of International Wireless Communications and Mobile Computing Conference (IWCMC), August 4-8, 2014. Article (CrossRef Link)

[11] Z. Li, Q. Liang, C. Xiuzhen, and T. Znati, "Outage performance evaluation over Rayleigh fading channels of WD networks," in Proc. of IEEE Innovative Smart Grid Technologies - Asia (ISGT Asia), 2012, pp. 1-5, May 21-24, 2012. Article (CrossRef Link)

[12] L. Xiaofeng, H. Pan, and P. Lio, "Offloading mobile data from cellular networks through peer-to-peer WiFi communication: A subscribe-and-send architecture," China Communications, vol. 10, no. 6, pp. 35-46, June, 2013. Article (CrossRef Link)

[13] E. C. Park, N. Kwak, S. K. Lee, J.K. Kim, and H. Kim, "Provisioning QoS for WiFi-enabled Portable Devices in Home Networks," KSII Transactions on Internet and Information Systems, vol. 5, no. 4, pp. 720-740, 2011. Article (CrossRef Link)

[14] R. Tang, J. Zhao, H. Qu, Z. Zhu, and Y. Zhang, "Joint Mode Selection and Resource Allocation for Mobile Relay-Aided Device-to-Device Communication,” KSII Transactions on Internet and Information Systems, vol. 10, no. 3, pp. 950-975, 2016. Article (CrossRef Link)

[15] W. H. Kuo, R. Kaliski, and H. Y. Wei, "A QoE-Based Link Adaptation Scheme for H.264/SVC Video Multicast Over IEEE 802.11," IEEE Transactions on Circuits and Systems for Video Technology, vol. 25, no. 5, pp. 812-826, May, 2015. Article (CrossRef Link)

[16] M. Jo, D. Araujo, T. Maksymyuk, A. Almeida, and T. F. Maciel, "Massive MIMO: Survey and Future Research Topics,” IET Communication, pp.1-26, 2016. Article (CrossRef Link)

[17] X. Ge, R. Zi, H. Wang, J. Zhang, and M. Jo, "Multi-user massive MIMO communication systems based on irregular antenna arrays," IEEE Transactions on Wireless Communications, vol. 15, no. 8, pp. 5287-5301, August, 2016. Article (CrossRef Link)

[18] IEEE 802.11n-Part 11: Wireless LAN Medium Access Control (MAC) and Physical Layer (PHY) Specifications: Enhancements for Higher Throughput, IEEE 802.11n-2009. Article (CrossRef Link)

[19] Wi-Fi Alliance, WiFi Protected Setup Specification v 1.0 h, December, 2006. Article (CrossRef Link)

[20] W. K. Edwards, "Discovery systems in ubiquitous computing," IEEE Pervasive Computing, vol. 5, no. 2, pp. 70-77, April-June, 2006. Article (CrossRef Link)

[21] https://www.youtube.com/user/ZombieSymmetry/videos. Article (CrossRef Link)

[22] V. Erceg, L. Schumacher, P. Kyritsi, et al., “TGn Channel Models," Version 4. IEEE 802.11-03/940r4, May, 2004. Article (CrossRef Link)

[23] G. Bianchi, "TGn Channel Models," IEEE P802.11 Wireless LANs, IEEE 802.11-03/940r4, 2004. Article (CrossRef Link)

[24] L. Schumacher, K. I. Pedersen, and P.E. Mogensen, "From antenna spacings to theoretical capacities - guidelines for simulating MIMO systems," in Proc. of PIMRC Conf., vol. 2, pp. 587-592, September, 2002. Article (CrossRef Link)

[25] V. J. Rhodes, "Path loss proposal for the IEEE 802.11 HTSG channel model Ad Hoc group," April 22, 2003. Article (CrossRef Link)

[26] J. B. Andersen, T. S. Rappaport, and S. Yoshida, "Propagation measurements and models for wireless communication channels," IEEE Communication. Magazine, pp. 42-49, January, 1995. Article (CrossRef Link)

[27] A. Perahia and R. Stacey, "Next Generation Wireless LANs - Throughput, Robustness, and Reliability in 802.11n,” Cambridge, 2008. Article (CrossRef Link) 
[28] Y. S. Cho, J. Kim, MIMO-OFDM wireless communications with MATLAB, John Wiley and Sons, 2010. Article (CrossRef Link)

[29] ITU-T SG12, “Definition of Quality of Experience,” COM12 - LS 62 - E, TD 109rev2 (PLEN/12), Geneva, Switzerland, January 2007. Article (CrossRef Link)

[30] I. H. Mkwawa, E. Jammeh and L. Sun, "Mapping of Received Signal Strength Indicator to QoE in VoIP applications over wlan," in Proc. of Fourth International Workshop on Quality of Multimedia Experience (QoMEX), Yarra Valley, VIC, pp. 156-157, 2012. Article (CrossRef Link)

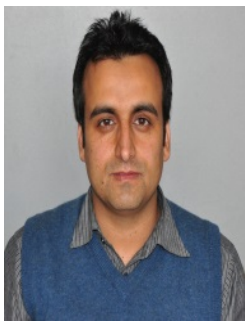

Gul Zameen Khan received a Bachelor degree in Computer Systems Engineering from University of Engineering and Technology, Peshawar, Pakistan in 2007 and a Master degree in Computer Engineering from Hanyang University South Korea in 2011. $\mathrm{He}$ is currently working as a researcher with the Intelligent Wireless Technology and Applications research group at Griffith University Australia.He has worked as a Lab Engineer in Ghulam Ishaq Khan Institute of Engineering Sciences and Technology Pakistan. In addition, he has worked as a lecturer in Sarhad University of IT Peshawar Pakistan and COMSATS Institute Abbottabad Pakistan. He has also worked as a Research Assistant at Wireless Networks Lab, Dongguk University, South Korea. His areas of interest are MAC and PHY layers analysis of 802.11, multicast in Wi-Fi Direct, and wireless sensor networks. Mr. Gul Zameen Khan is a member of IEEE and Institute for Integrated and Intelligent Systems, Griffith University Australia. He is also a professional member of Pakistan Engineering Council

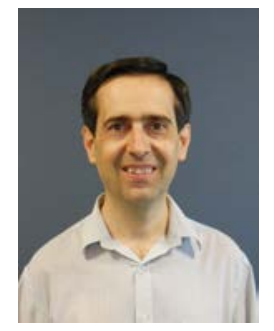

Dr. Ruben Gonzalez received a B.E. and Ph.D. from the University of Technology, Sydney (UTS) and is currently a senior Lecturer in the School of ICT, at Griffith University Australia. He is also the director of the Bachelor of Computer Science at Griffith University. Previously he was founder and CTO of ActiveSky Inc, a wireless media technology company and has also held research positions at Wollongong University, OTC Ltd Research Labs and UTS. He has also held software engineering positions at various private enterprises. Dr. Ruben Gonzalez is a member of the Institute for Integrated and Intelligent Systems, Griffith University Australia. He has over 80 refereed publications and a number of patents

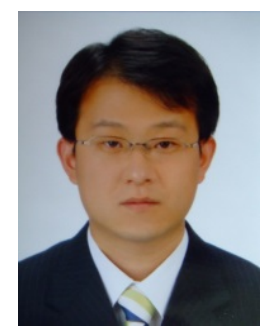

Prof. Eun-Chan Park received the B.S., M.S., and Ph.D. degrees from the School of Electrical Engineering and Computer Science, Seoul National University, Seoul, Korea, in 1999, 2001, and 2006, respectively. He worked for Samsung Electronics, Korea, as a senior engineer from 2006 to 2008. He is currently an Associate Professor in the Department of Information and Communication Engineering, Dongguk University-Seoul, Korea. He is also the head of the Wireless Networks Lab, at Dongguk University South Korea. His research interests include performance analysis, resource allocation, quality of service, congestion control, and cross-layer optimization in wired and wireless networks. Dr. Eun-Chan Park is a member of IEEE Communications Society. 